\title{
Uso de modelos matemáticos para la descripción del crecimiento de tumores cancerosos
}

\author{
Jair Zapata Peña. J', Alba Cristina Ortiz P2 \\ 1. Universidad Antonio Nariño, Universidad Pedagógica Nacional, Bogotá. \\ 2. Universidad La Gran Colombia, Bogotá.
}

Correspondencia: jzapata25@gmail.com

Recibido: 05-08-10 / Aceptado: 28-11-10

\begin{abstract}
Resumen
La producción de tumores cancerosos o tumorgénesis ha sido estudiada desde principios del siglo XX por matemáticos y físicos interesados en aplicaciones biológicas. En este trabajo se plantean diversos modelos que utilizan ecuaciones diferenciales ordinarias, ecuaciones diferenciales parciales, modelos estocásticos discretos, estadísticos y de análisis numérico para describir el crecimiento de tumores cancerosos. Se muestra un análisis comparativo entre estos modelos matemáticos, estableciendo particularidades y limitaciones debido a la especificidad de las poblaciones cancerosas. Se amplía un estudio del modelo de competencia por nutrientes utilizando una simulación computacional, donde se muestran resultados gráficos de las simulaciones para poblaciones de células cancerosas y muertas. Palabras clave: células cancerosas, modelos matemáticos, competencia por nutrientes, simulación computacional.
\end{abstract}

\section{Abstract \\ Use mathematical models to describe the growth of cancerous tumors}

The production of cancerous tumors or tumorigenesis has been studied from principles of the twentieth century by mathematicians and physicists interested in biological applications. In this paper we discuss various models using ordinary differential equations, partial differential equations, discrete stochastic models, statistical-cal and numerical analysis to describe the growth of cancerous tumors. Shows a comparison between these mathematical models, setting characteristics and limitations due to the specific cancer populations. It extends a model study competition for nutrients using a computer simulation, which shows graphical results of simulations for populations of cancer cells and dead. Keywords: cancer cells, mathematical models, competition for nutrients, computer simulation. 


\section{Introducción}

Aunque la evolución de un sistema cancerígeno está limitada y condicionada a parámetros químicos y biológicos, su diseminación a través de los tejidos y órganos contaminados obedece a diversas reglas deterministas o estocásticas, las cuales se pueden modelar utilizando herramientas matemáticas y condicionamientos físicos (1). La producción de tumores cancerosos o tumorgénesis ha sido estudiada desde principios del siglo XX por matemáticos interesados en aplicaciones biológicas (2). Se plantean diversos modelos que utilizan ecuaciones diferenciales ordinarias (EDO), ecuaciones diferenciales parciales (EDP), modelos estocásticos discretos, estadísticos y de análisis numérico.

Existen trabajos alrededor de este tema que muestran, por un lado, resultados referidos a tratamientos particulares con modelos no generalizados, y por otro, estructuras funcionales que permiten la aplicabilidad a diversos tipos de cáncer. Sin embargo, hoy en día se carece de un modelo que proporcione una predicción y caracterización del comportamiento para el crecimiento de tumores cancerosos en sus múltiples formas y para cualquier tipo de población, teniendo en cuenta, que los modelos existentes funcionan bajo condiciones ideales y con poblaciones específicas (3), científicos de diferentes áreas han desarrollado modelos para el tratamiento de la dinámica del crecimiento tumoral. Modelos provenientes de diversos campos como la matemática aplicada, la estadística, la ciencia computacional, la ciencia de materiales, la mecánica de fluidos, la dinámica poblacional, la evolución y la teoría del juego han sido desarrollados (4).

En este trabajo se muestra un análisis comparativo entre estos modelos matemáticos estableciendo particularidades y limitaciones de generalización debido a la especificidad de las poblaciones cancerosas. Se amplía un estudio del modelo de competencia por nutrientes utilizando una simulación computacional, donde se muestran resultados gráficos de las simulaciones para poblaciones de células cancerosas y muertas.

\section{Modelos matemáticos}

Ecuaciones diferenciales ordinarias (EDO): este modelo supone una población de células cancerosas con igual potencial de crecimiento. Se aplica a problemas de estabilidad y permanencia de sistemas donde se tiene una población biológica compitiendo por recursos. La EDO describe la competición entre organismos como un cambio en la función de saturación. El número de células en un tiempo $t$ depende de la tasa de crecimiento y está asociado con la capacidad de transporte, que es el tamaño máximo de la población que se puede alcanzar, definida por el nutriente suplido y las restricciones espaciales.

La solución a esta ecuación es la curva sigmoidal. Una aplicación de este modelo fue introducido por Manfred Eigen en 1971 como un camino para modelar la evolución dinámica de moléculas únicas de ARN en experimentos de laboratorio, conocido como el modelo de las cuasiespecies. Luego se extendió este modelo al tratamiento y evolución de virus, bacterias y modelos simples del sistema inmune (2). El modelo de Eigen supone una población heterogénea de células $i$ que compiten entre sí y rodean las células saludables en busca de nutrientes, oxigeno y espacio. En este modelo el número total de células del sistema se supone constante y se permite el desencadenamiento de mutaciones que generan divisiones celulares a una razón determinada para cada tipo de célula, es decir, una célula de tipo $i$ solo puede mutar en una del tipo $i+1$ (5).

Ecuaciones diferenciales parciales: este método es una herramienta muy usada en el estudio del crecimiento de tumores y la forma en que se difunden sobre los tejidos que los rodean (6-7). En la utilización de estas ecuaciones se supone un sistema descrito bajo un comportamiento mecánico, donde el sistema puede ser un fluido o una mezcla entre liquido y sólido (los fluidos son normalmente los nutrientes). Se presentan fenómenos de difusión y transporte de nutrientes teniendo en cuenta efectos de concentración, tamaño y velocidad de células. En este modelo se aplican condiciones iniciales y de frontera que están relacionadas con el tamaño, la permeabilidad del medio, la geometría y las dimensiones del sistema. Anderson y Chaplain 
plantearon una EDP para describir la dinámica de la densidad de células endoteliales (EC) que migran a través de un tumor y forman estructuras neovasculares en respuesta a una señal química especifica conocida como factor angiogénico del tumor (TAF). Ellos proponen que si $n(x, t)$ es la densidad EC, entonces la migración esta descrita por

$$
\dot{n}=D \nabla^{2} n-\nabla(\chi(c) n \nabla c)+g(n, c)
$$

Donde $D$ y $X^{(c)}$ son el coeficiente de difusión y el parámetro quimio-táctico respectivamente, $c(x, t)$ es la concentración de TAF químico especifico responsable de la quimiotaxis y $g(n, c)$ es la función de proliferación. Para el caso simple la señal química se puede suponer como estacionaria (independiente del tiempo), si no es estacionaria esta debe satisfacer la ecuación diferencial

$$
\dot{c}=D c \nabla^{2} n+v(c, n),(\mathrm{b})
$$

Con $\mathbf{v}(\mathbf{c}, \mathbf{n})$ como la función específica de producción de la señal química (8).

Modelo discreto, autómata celular: la estructura del modelo está basada en una región del espacio discreta (rejilla cuadrada), donde coexisten unas determinadas especies celulares. La dinámica de crecimiento está definida por algunas reglas de interacción a lo largo de los nodos y entre los vecinos de cada celda. Las reglas pueden ser determínisticas o estocásticas, estas últimas son dictaminadas por procesos aleatorios con inclusión de probabilidades. Cada punto de la rejilla puede representar una célula individual o un grupo de células. En una red de autómatas el estado de una célula dentro de la región depende de las reglas definidas, del estado de los vecinos y de su propio estado en la generación anterior.

Un ejemplo básico de autómata celular es una rejilla en dos dimensiones, con puntos nodales $x_{i, j}$ donde $i$ y $j$ son las dimensiones de la cuadricula. Se empieza con una población inicial, que se actualiza en cada paso de tiempo bajo unas determinadas reglas de muerte y reproducción. Los modelos de autómata celular usados para describir sistemas reales son más complejos y tienen en cuenta factores biológicos que caracterizan la difusividad y crecimiento del tumor. El modelo de autómata celular en tres dimensiones describe el crecimiento de un tumor cerebral. En él se incorporan dos tipos de células proliferativas y no proliferativas, que requieren para su evolución de dos cuadriculas una isotrópica y otra adaptable (9-10).

Modelo estocástico: muchos fenómenos biológicos son gobernados por variables aleatorias, y requieren de un modelo que involucre procesos al azar. Según este modelo no se puede predecir con exactitud el estado de un sistema en un tiempo dado pero se logran predecir ciertas tendencias bajo las cuales este sistema evoluciona paulatinamente de tal manera que para experimentos repetidos se encuentran resultados similares pero no idénticos. El proceso de Moran es un modelo estocástico simple el cual describe el comportamiento de una población basado en una dinámica de nacimiento y muerte. Aquí se considera una población de tamaño $\mathrm{N}$, con células sanas $\mathrm{y}$ mutadas. En cada paso de tiempo un individuo A es seleccionado aleatoriamente para su reproducción. Debido a esto, otro individuo $B$ es seleccionado para morir. La descendencia de A reemplaza a la célula muerta $\mathrm{B}$, manteniendo a $\mathrm{N}$ constante.

Así pues el proceso de Moran describe la evolución estocástica de una población finita de tamaño constante. Se supone que todos los individuos residentes son idénticos y se introduce un mutante que tiene un fitness $r$ (el termino fitness, se refiere a la aptitud en el contexto biológico, que es el valor adaptativo que tiene un organismo para poder pasar los genes a futuras generaciones y que sus descendientes puedan nacer y sobrevivir), mientras que el de los residentes es 1, es decir, que los mutantes pueden ser menos o más aptos para sobrevivir que sus predecesores. Los únicos estados estables posibles en el proceso de Moran son o todos mutantes o todos residentes. Komarova describe comportamientos celulares de organismos adultos como un modelo simple que tiene como característica una población constante (11). Otros modelos proponen además poblaciones en continuo crecimiento o decadencia continua hasta su extinción, como es el caso del proceso one-hit o el two-hit (12-13). 
Modelo estadístico: este modelo es utilizado en procesos donde las múltiples etapas de la carcinogénesis se presentan como una serie de procesos estocásticos con eventos mutacionales y de expansión de clones. Para este modelo se proponen una serie de variables como tasas de mutación, tasas promedio de expansión de clones por etapa y número de etapas. La probabilidad de desarrollo de cáncer para una cierta edad es calculada, usualmente por simulaciones numéricas, como una función de todos los parámetros desconocidos. El resultado de tales cálculos para cada conjunto de parámetros es comparado con los datos existentes de la incidencia de cáncer, donde se identifica el conjunto de parámetros que mejor se ajuste. Trabajos en los que se analizan curvas de incidencia y muerte con la edad muestran diferentes curvas con las fluctuaciones estadísticas de las muertes a causa del cáncer por año, se destacan las diferencias entre el cáncer a edades tempranas y después de los cuarenta años (14-15).

\section{Modelo de competencia por nutrientes}

Scalerandi y colaboradores desarrollaron un modelo para el crecimiento del cáncer basado en la competencia por nutrientes. Este modelo supone una población celular condicionada en una región del espacio discreta (16). Se formulan una serie de reglas a nivel celular, las cuales son implementadas con ecuaciones diferenciales no lineales. Las ecuaciones diferenciales son discretas y solucionadas usando simulaciones numéricas. Se considera una región de tejido completamente sana. El tejido se representa por una red cuadrada, donde cada punto nodal $i, j$ representa un elemento de volumen que contiene varias células y moléculas de nutrientes. Se coloca una semilla de cáncer en el centro de la red. Luego de un paso de tiempo se supone la coexistencia de tres poblaciones, células cancerosas, células sanas y células muertas, dentro de los nodos que conforman la red. La evolución de esta concentración de células es descrita por ecuaciones diferenciales de primer orden en función del tiempo, que están sujetas a unas determinadas reglas de crecimiento. Estas reglas dependen de la disponibilidad de nutrientes en las cercanías del tumor, de las tasas de absorción y difusión de nutrientes, así como de las cantidades de células muertas, sanas y cancerígenas.

\section{Reglas para el crecimiento de tumores}

Para las reglas de crecimiento se supone la coexistencia de tres poblaciones: células cancerosas $c(\vec{r}, t)$, células sanas $h(\vec{r}, t)$ y células muertas $d(\vec{r}, t)$ , dentro de los nodos que conforman la red. En el instante inicial $t=0$ se coloca una semilla de cáncer dentro de una región con población celular sana. El sistema evoluciona y la distribución de las células que ocupan un nodo varía con el tiempo, pero se supone que la población total por nodo se conserva y está normalizada, de tal forma que:

$$
h(\vec{r}, t)+c(\vec{r}, t)+d(\vec{r}, t)=1 \text {. (c) }
$$

La cual se cumple en todo momento y en toda la red. El modelo propone para la evolución en el tiempo de estas tres poblaciones una serie de ecuaciones de diferencias de primer orden, que requieren de una solución numérica. Estas ecuaciones se escribirán en adelante de forma discreta omitiendo por brevedad la dependencia con el tiempo. Se describen a continuación las reglas para el crecimiento de tumores cancerosos según este modelo: alimentación, consumo, muerte, mitosis, migración.

Alimentación: los nutrientes libres $p(\vec{r})$ son transformados en nutrientes ligados $q(\vec{r})$ con una tasa de absorción $\tilde{\gamma}$ igual a

$$
\tilde{\gamma}(\vec{r})=\tilde{\gamma}_{a s}\left(1-e^{-\Gamma p(\vec{r})}\right),(\mathrm{d})
$$

donde $\Gamma$ es un parámetro de afinidad y $\widetilde{\gamma}_{\alpha}$ representa el valor de saturación de la tasa de absorción para valores grandes de nutriente libre, $p(\vec{r})$.

Consumo: los nutrientes ligados $q(\vec{r})$ son consumidos por las células cancerosas con una tasa de

$$
\beta(\vec{r})=\beta_{a s}\left(1-e^{-q(\vec{r}) / c(\vec{r})}\right),(\mathrm{e})
$$

Donde el término $c(\vec{r})$ ha sido incluido en el exponente porque cada célula puede consumir únicamente su propio nutriente ligado.

Muerte: la mortalidad de células depende de la concentración de nutriente ligado, que es necesario 
para el funcionamiento de las células, la escasez de éste produce la muerte celular. Si la cantidad promedio de nutriente ligado por célula cancerosa $q(\vec{r}) / c(\vec{r})$, en un nodo $\vec{r}$, cae por debajo del umbral de muerte $Q_{D}$, un número de células cancerosas $r_{D} c(\vec{r})$ mueren de hambre en el siguiente paso.

Mitosis: una alta concentración de nutriente ligado puede ocasionar división celular. Cuando la cantidad promedio de nutriente ligado por célula cancerosa $q(\vec{r}) / c(\vec{r})$ excede el umbral de mitosis, $Q_{M}\left(Q_{M}>Q_{D}\right)$ , una fracción $m(\vec{r})$ de células sanas es transformada en células cancerosas. Esta fracción está dada por,

$m(\vec{r})=h(\vec{r})+\left[r_{M} c(\vec{r})-h(\vec{r})\right] \Theta\left[r_{M} c(\vec{r})-h(\vec{r})\right]$.

Aquí $\Theta(x)$ es la función escalón de Heaviside, la cual verifica cuando la cantidad promedio de nutriente ligado es menor que el umbral de muerte y cuando la cantidad promedio de nutriente ligado se hace mayor que el umbral de mitosis. $r_{M}$ es una constante.

Migración: si los nutrientes libres son pocos, las células cancerosas tienden a migrar a los nodos vecinos más cercanos. Asumiendo que si la cantidad promedio de nutriente libre por célula cancerosa en un nodo $\vec{r}$, es $p(\vec{r}) / c(\vec{r})<P_{D}$, las células cancerosas migraran a los nodos vecinos con un coeficiente de migración $\tilde{\alpha}$ el cual depende del tipo de nutriente. Donde $P_{D}$ es la cantidad mínima de nutriente libre por célula cancerosa, existente en los alrededores, para el cual no se produce migración.

\section{Materiales y métodos}

Las funciones vitales que tienen lugar en el interior de las células, a nivel microscópico, tales como la síntesis de proteínas, la transmisión de información genética y demás, involucran procesos de alta complejidad para la realización de análisis matemáticos y caracterizaciones físicas. Sin embargo, cuando se estudian los tejidos a una escala mayor como la mesoscópica existen algunas estructuras biológicas sobre las cuales subyace la idea de un modelo de coexistencia celular.

Este modelo obedece dinámicas de movilidad, reproducción y muerte que están sujetas a procesos de supervivencia celular, en los cuales las diferentes poblaciones que conviven en un tejido compiten por alimento y espacio. Teniendo en cuenta que una célula no tiene sentido de su posición en el tejido, sino que solo responde a señales y estímulos provenientes de su entorno local (3), el análisis mesoscópico desarrollado en este trabajo pretende describir el comportamiento celular a esta escala, utilizando el modelo matemático de competencia por nutrientes.

Para la realización de las simulaciones computacionales se discretizaron las ecuaciones diferenciales que propone el modelo, utilizando diferencias finitas; luego de ello se desarrolló un programa en $\mathrm{C}_{++}$, con programación orientado a objetos. Los resultados numéricos que arrojaban las simulaciones se visualizaron a través de graficas generadas en Matlab 7.0, para su posterior análisis, discusión y convalidación con expertos de la Universidades de Puerto Rico y Córdoba Argentina (17, 18).

\section{Resultados}

En este trabajo se amplía un estudio del modelo de competencia por nutrientes. Se considera una red de tejido de $10 n$ x 10 . La red se selecciona pequeña ya que se debe garantizar mantener el tejido dotado de los nutrientes y oxigeno necesarios para que las células puedan desarrollar sus funciones biológicas básicas. El diámetro promedio de las células de los mamíferos es aproximadamente de $10 \mu \mathrm{m}$. Teniendo en cuenta que el modelo utilizado está basado en concentraciones celulares resulta apropiado suponer la red del tejido de 100 x 100 nodos. Así cada nodo tendrá una dimensión de $100 \mu m \times 100 \mu m$. Considerando el tamaño celular cada nodo contendrá 100 células. La discretización espacial estará dada por $\Delta=100 \mu \mathrm{m}$. Para la discretización temporal se debe garantizar la estabilidad de la red de acuerdo con...

$$
\tau \leq \frac{\Delta^{2}}{2}
$$

para no tener problemas en la discretización de las derivadas donde se supone que $\Delta t=8 h$, es decir que 3 pasos de simulación corresponden a un día o que $t=1000$ pasos corresponden aproximadamente a un 

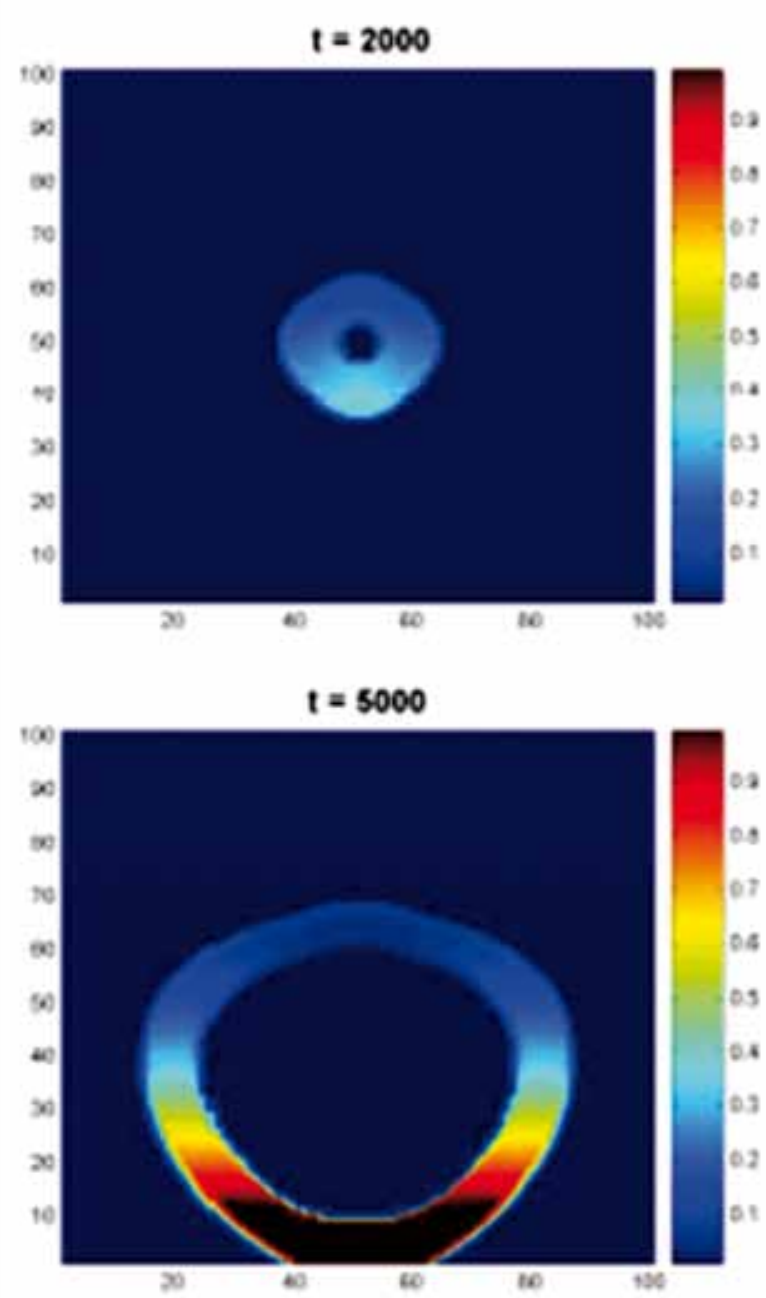

Figura 1. Morfología del crecimiento de células cancerosas. Tumor en diferentes iteraciones de tiempo. Parámetros tomados de Komarova y colaboradores, mayor concentración cancerosa color rojo disminuyendo la densidad hacia el color azul.

año de tiempo real (17). Este es un valor estimado para un correspondiente crecimiento cancerígeno cercano a los 5 años para producirse la intravasación (18).

La Figura 1 muestra el comportamiento de las células cancerosas para el crecimiento de un tumor. Se observa que a partir de una semilla en el centro de la red se genera el crecimiento del tumor canceroso. El tumor inicia como una masa uniforme y concentrada, la cual presenta una baja densidad de células cancerosas (azul) en la región superior, la actividad cancerosa aumenta hacia la parte baja del tumor, región roja, orientado hacia la parte inferior de la red donde se encuentra ubicada la vena, por donde circulan los nutrientes. Se genera una zona denominada núcleo necrótico, región azul oscuro,
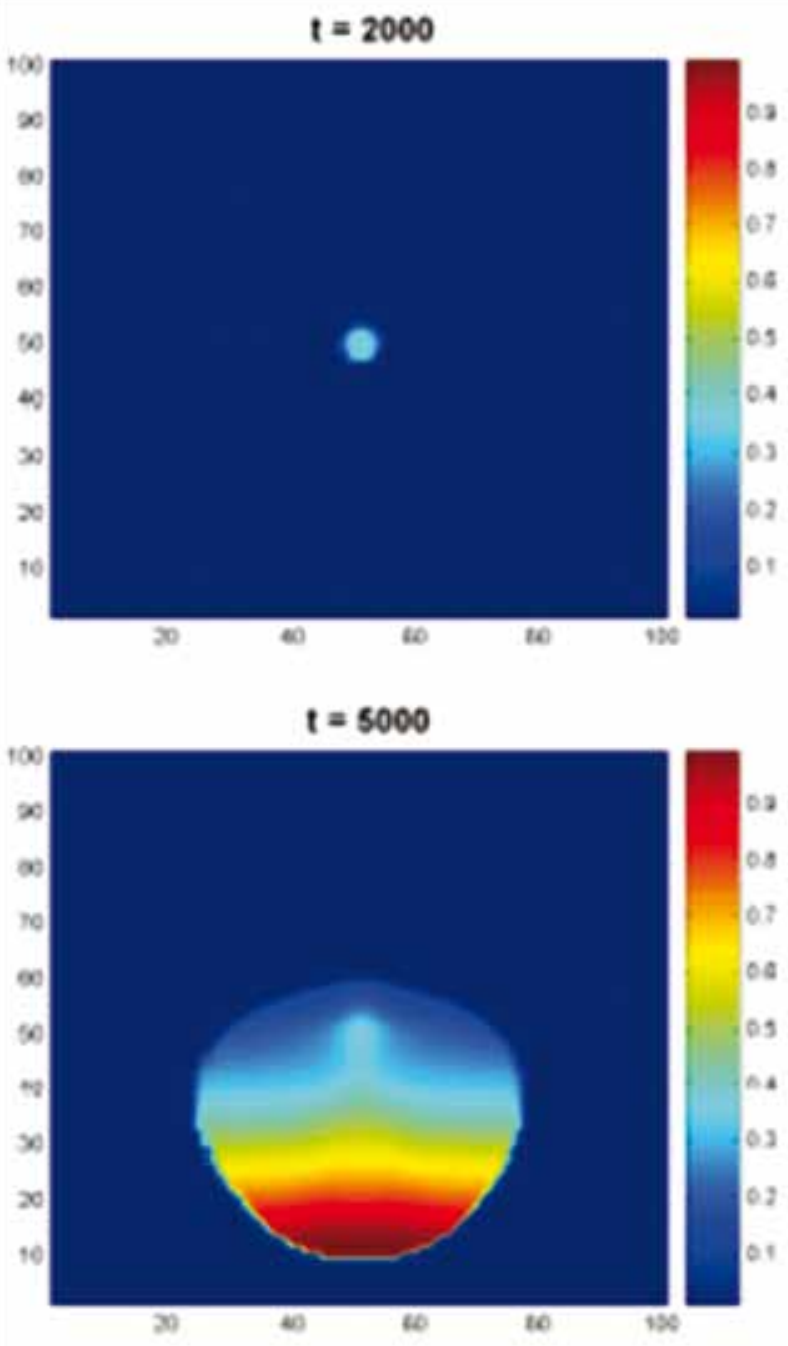

Figura 2. Morfología de las células muertas. La región azul oscuro está completamente sana y los colores rojos, amarillos y azules claros representan las regiones con mayor número de células muertas del tumor.

ubicada en la parte central y caracterizada por contener las células muertas del tumor. Estas células son originadas a partir de las cancerígenas que no se pueden reproducir o mueren por falta de alimento y espacio.

En la Figura 2 se muestra el comportamiento de la concentración de células muertas. Se aprecia en estas imágenes como este crecimiento se origina desde la parte central y se expande de forma maciza hacia el exterior, generando lo que anteriormente se denomino núcleo necrótico. Es importante notar que las simulaciones muestran una aparición de células muertas solamente en la región central del tumor. Este comportamiento se da porque la actividad celular, 
como consumo de nutrientes y búsqueda de espacio, se desarrolla principalmente en la periferia del tumor que es la zona más agresiva (19-20). Las células que van quedando en la parte interna comienzan a morir por falta de condiciones suficientes para cumplir sus funciones vitales.

\section{Discusión}

En el modelo de competencia por nutrientes se encontraron comportamientos particulares asociados con la disponibilidad de nutrientes y de espacio, evidenciándose que las células cancerosas compiten por estos dos. El comportamiento del modelo refleja que la proliferación cancerosa depende estrictamente de cuatro parámetros: la movilidad de las células cancerosas $\widetilde{\alpha}$, la capacidad de absorción de nutrientes $\widetilde{\gamma}_{a s}$, la capacidad para consumir nutrientes $\beta_{a s}$ y la disposición del nutriente libre en el tejido $p(\vec{r})$.

Las simulaciones muestran que las células muertas se ubican esencialmente en la región central del tumor, debido a que la actividad celular, como consumo de nutrientes y búsqueda de espacio, se desarrolla principalmente en la periferia del tumor que es la zona más agresiva y con mayor actividad. Este comportamiento es similar a lo encontrado en el trabajo de Brú (20), el cual explicita como un factor predominante privilegia la posición que brinda la región externa del tumor, debido a su proximidad y mayor interacción con los nutrientes y el espacio libre para la proliferación de las células cancerosas.

Se encuentra además algunas similitudes entre el modelo de competencia por nutrientes con el modelo Autowaves para el crecimiento de los tumores invasivos (21), que tiene en cuenta la división celular, la muerte y la movilidad. Este modelo incluye además la densidad celular local y la distribución de la concentración de nutrientes, que se podría relacionar con la distribución de nutrientes libres $p(\vec{r})$ usados en el modelo de competencia por nutrientes. Cabe destacar que el modelo Autowaves no tiene especificidad en las tasas de absorción, en el consumo de nutrientes y migración, como si lo hace el utilizado en este trabajo de competencia por nutrientes.
Este modelo refleja consistencia y validez ya que permite predecir diferentes tasas de crecimiento y morfologías tumorales al variar algunos de sus parámetros, como la movilidad, el consumo y la absorción de nutrientes. Si el parámetro $\widetilde{\gamma}_{\text {as }}$ se hace grande el nutriente libre se consumirá más rápido ocasionando migración celular. Por otro lado si $\beta_{a s}<\widetilde{\gamma}_{a s}$ las células cancerosas aumentan su reproducción ya que disponen de una mayor concentración de nutriente ligado; esto ocasiona una posterior reducción de $p(\vec{r})$ en la región, lo que origina la migración celular. El desarrollo de este modelo es consecuente con las reglas biológicas que involucran dinámicas de movilidad y crecimiento, en donde las células compiten por espacio y alimento con células de la misma clase, de otras clases o vecinas.

Otra fortaleza del modelo es la capacidad que tiene de predecir tiempos de vida de algunos órganos afectados, así como de ofrecer criterios temporales para pronosticar procesos de metástasis, los cuales pueden ser de gran utilidad en la aplicación medica real (17).

Los resultados obtenidos en este conllevan a considerar que el método EDO presenta como ventaja la simplicidad en el desarrollo, pero es poco detallado excluyendo eventos aleatorios, lo que implica una aplicabilidad restringida. El modelaje con EDP describe dinámicamente variaciones espaciales del sistema que son más convincentes biológicamente que las encontradas con las EDO, pero el tratamiento físico no se puede idealizar ya que faltan parámetros biológicos y la intervención de los efectos estocásticos. El modelaje discreto, muestra una nueva clase de comportamiento que no puede ser visto en el modelo continuo, permitiendo rastrear celdas individuales reproduciendo las dinámicas que surgen de la estructura como la vascularización de tumores. Esta aproximación es netamente numérica dificultando el análisis del modelo y la generalización del mismo para diversas tendencias.

El modelaje computacional se muestra como una herramienta esencial en el tratamiento de las dinámicas de crecimiento, siendo ésta la forma más eficiente de estudiar y visualizar el comportamiento de poblaciones que obedecen estructuras discretas y fenómenos de aleatoriedad. Con el modelo estudiado 
de competencia por nutrientes se analizaron diversas morfologías de crecimiento, estudiando y evaluando sistemáticamente el comportamiento y la dinámica tumoral al variar los parámetros que regulan el modelo, como absorción y consumo de nutrientes, migración, mitosis y muerte celular. En este proceso se encontraron comportamientos particulares asociados con la disponibilidad de nutrientes y de espacio, resaltándose que las células cancerosas compiten por estos dos.

\section{Agradecimientos}

Departamento de Física de la Universidad de Puerto Rico-Mayagüez.

\section{Referencias}

1. Kolev M, Zubik-Kowal B. Numerical Solutions for a Model of Tissue Invasion and Migration of Tumour Cells. Comput Math Methods Med. 2011;2011:452320.

2. Solé RV, Deisboeck TS. An error catastrophe in cancer?. J Theor Biol. 2004;228:47-54.

3. Menchón S.A. Modelado de diversas etapas del crecimiento del cáncer y de algunas terapias antitumorales. Córdoba. Tesis Doctoral (Doctorado en física), Universidad Nacional de Córdoba, Argentina, Departamento de física. 2007.

4. Wodarz D, Komarova NL. Computational Biology Of Cancer: Lecture Notes And Mathematical Modeling. Ed. World Scientific, Singapore. 2005. p.13-26.

5. Sotolongo-Costa O, Morales L, Rodríguez D, Antoranz JC, Chacon M. Behavior of tumor under nonostationary therapy. Physical D. 2003;178:242-253.

6. Kim Y, Friedman A. Interaction of tumor with its micro-environment: A mathematical model. Math Biol. 2010;72:1029-1068.
7. Doumic-Jauffret M, Kim PS, Perthame B. Stability analysis of a simplified yet complete model for chronic myelogenous leukemia. Bull Math Biol. 2010;72:1732-1759.

8. Anderson AR, Chaplain MA. Continuous and discrete mathematical models of tumor-induced angiogenesis. Bull Math Biol. 1998;60:857899.

9. Kansal AR, Torquato S, Harsh GR IV, Chiocca EA, Deisboeck TS. Simulated brain tumor growth dynamics using a three-dimensional cellular automaton. J Theo Biol. 2000;203:367-382.

10. Azuaje F. Computational discrete models of tissue growth and regeneration. Brief Bioinform. 2011;12:64-77.

11. Komarova NL, Sengupta A, Nowak MA. Mutation-selection networks of cancer initiation: tumor suppressor genes and chromosomal instability. J Theor Biol. 2003;223:433-450.

12. Knudson AG. Hereditary cancer: two hits revisited. J Cancer Res Clin Oncol. 1996;122:135-140.

13. Knudson AG. Mutation and cancer: statistical study of retinoblastoma. En: Proc Natl Acad Sci USA. 1971;68:820-823.

14. Frank SA. Age-specific acceleration of cancer. Curr Biol. 2004; $14: 242-246$

15 Krewski D, Zielinski JM, Hazelton WD, Garner MJ, Moolgavkar $\mathrm{SH}$. The use of biologically based cancer risk models in radiation epidemiology. Radiat Prot Dosimetry. 2003;104:367-376.

16. Pescarmona GP, Scalerandi M, Delsanto PP, Condat CA. Nutrient competition as a determinant for cancer growth. Med Hypotheses. 1999;53:497-503.

17. Zapata J.Jair M. Simulaciones por computadora de un modelo espaciotemporal para la interacción del sistema inmunológico y los tumores cancerosos. Dissertations \& Theses. 2009;1:76-80.

18. Menchón SA, Ramos RA, Condat CA. . Modeling subspecies and tumor-immune system interaction: Step towards understanding therapy. Physica A. 2007;386:713-719.

19. Brú A, Pastor JM, Brú I, Melle S, Berenguer C. Super rough dynamics on tumor growth. Phys Rev Lett. 1998;18:4008- 4011.

20. Brú A, Albertos S, López García-Asenjo JA, Brú I. Pinning of tumoral growth by enhancement of the immune response. Phys Rev Lett. 2004;92:238101-238104.

21. Kolobov AV, Gubernov VV, Polezhaev AA. Autowaves in a model of growth of an invasive tumor. Biofizika. 2009;54:334-342. 